\title{
Hypoglycaemia monitoring in a medical receiving ward
}

\author{
Ryan Ellis
}

NHS, Scotland, UK

\begin{abstract}
It has been suggested that current care for diabetes inpatients remains inadequate and that greater attention is required for high quality management. In this project the aspect of hypoglycaemia was studied in a busy medical receiving ward at the Glasgow Royal Infirmary. A large proportion of inpatients have diabetes and episodes of hypoglycaemia experienced by this population can delay discharge and indeed be detrimental to health. Thus it is important from both an organisational and patient perspective to manage this population well. In this project BM machine data was analysed to identify patients who were hypoglycaemic. These patients were then tracked down to study the subsequent management and compared this against recommended guidance. Following this an intervention was made to promote identification, management, documentation, and prevention of hypoglycaemia. This was deliberately a simple intervention involving discussions with staff and provision of basic documented guidance next to every BM machine. In the first phase 17 patients were identified and in a second and third phase 16 patients each time were further identified. Patients in the study were both type 1 and type 2 diabetics. Initial results in phase I were compared to results in phase II and III respectively. This intervention produced significant improvements in management with correct monitoring of low BMs (i.e. upon identification of low BM repeat within 1 hour) improving from $47 \%$ to $100 \%$ (for Phase II and III). Also, recording of preventative measures of hypoglycaemia improved from $35 \%$ to $88 \%$ and $94 \%$ with an improvement from $24 \%$ to $69 \%$ and $75 \%$ in recording of treatment given if needed. In conclusion, the study successfully demonstrated that simple measures can significantly improve the quality care of inpatient diabetic patients in relation to hypoglycaemia management.
\end{abstract}

\section{Problem}

ThinkGlucose, an NHS institute programme, was set up with the aim to support hospital trusts provide consistent, effective, and proactive inpatient care for patients with diabetes. The campaign states that current care for diabetes inpatients remains inadequate in the NHS resulting in longer admissions and more complications. With, at any one time, $10-25 \%$ of inpatients having diabetes this represents a failure to a large proportion of patients[1].

Furthermore, The National Institute for Health and Care

Excellence's (NICE) quality statement for inpatient diabetic care states that patients should be cared for by a "proportion of staff on inpatient wards who are appropriately trained to care for people with diabetes"[2]. To enable this excellence in care for such a great proportion of patients it is therefore necessary that all ward staff, from nurses to support workers and students, are aware of the requirements for good inpatient care of diabetes. This includes the ability to adequately identify an episode of hypoglycaemia and in doing so enable correct management and monitoring. The aim of this project was to focus on this care in regard to hypoglycaemia in a NHS Scotland acute medical receiving unit.

\section{Background}

Hypoglycaemia is a complication of diabetes that is common to both type 1 and insulin treated type 2 patients. Indeed, hypoglycaemia can also be caused by sulphonylureas e.g. gliclazide and possibly glitazones e.g. rosiglitazone which type 2 patients may additionally be prescribed. There is general appreciation of the need for strict outpatient management of glycaemic control in diabetes patients. However there is also now increasing evidence for the need for tight inpatient control[3].

Hypoglycaemia rightly constitutes a medical emergency that if long lasting and severe can lead to transient and even permanent cerebral damage[4]. Hypoglycaemia can be common in hospitalised patients with reports on the incidence of inpatient hypoglycaemia ranging up to $20 \%$ depending on population[5-7]. Such hypoglycaemic incidences have been found to be associated with poorer outcomes and increased mortality with suggestions that episodes can act as markers for more severe illness[8]. The elderly inpatients have also been found to be particularly at risk due to renal failure and cognitive impairment[9]. Moreover, in addition to this one study also demonstrated that inpatients with diabetes that experience hypoglycaemic episodes are more likely to have increased lengths of stay and greater rates of readmission putting further pressure on inpatient care[10]. However, if detected early and correctly hypoglycaemia can be easily and quickly reversed thus highlighting the importance of good monitoring and the need for strategies to prevent hypoglycaemia to be instituted.

Further to this is the importance of recognition of hypoglycaemic events. Symptoms of hypoglycaemia can be generally divided into two groups: adrenergic (effects of rapidly changing glucose levels) and neuroglycopenic (low central nervous system glucose). The presentation of adrenergic symptoms will precede neurobehavioral thus acting as an early warning system for ward staff.

These adrenergic signs and symptoms, that ward staff should be aware of, include anxiety, shakiness, dizziness, irritability, pallor, and tachycardia[11]. These signs and symptoms should be recognised to enable early treatment with simple carbohydrates or IV options (such as dextrose) and regular monitoring to prevent 
repeated hypoglycaemic episodes. This early recognition and intervention is important to prevent development of neuroglycopenic signs resulting in deterioration in central nervous system function. This if severe can degenerate to focal seizures and deep coma[12].

This monitoring and early recognition is increasingly important for hospital inpatients due to various factors that can disrupt an individual's own ability to manage and regulate their diabetes control. This includes disruptions to daily routine, altered appetite and/or mobility, alteration/additions to medications, and the pathophysiological effects of the actual presenting illness e.g. sepsis[13]. Furthermore, those patients who usually keep their diabetes tightly controlled, but lose control while unwell and in a different environment, may have problems recognising hypoglycaemic events and are at greater risk of repeated episodes[14]. Thus, this combination of diabetic inpatients having poorer control, less ability to recognise hypoglycaemic episodes and more likely to have repeated episodes emphasises the importance of good monitoring, early recognition, and intervention and need for strict follow up of any hypoglycaemic episodes in a timely manner to prevent repeated events.

\section{Baseline measurement}

Diabetes UK defines hypoglycaemia as the "medical term for low blood glucose level - that is blood glucose level less than 4 $\mathrm{mmol} / \mathrm{l} " 15$. This was thus the value taken for the project in which BM machines, used on the medical receiving wards at Glasgow Royal Infirmary, were scanned for patients with low blood glucose. On identifying a hypoglycaemic episode the patient was tracked down and the notes read for information on how the episode was recorded and managed. Guidance states that upon discovering a hypoglycaemic episode the BM should be rechecked within the hour. This was the target used for inpatient management of hypoglycaemia in diabetic patients. Additional information such as recording symptoms, management, and strategies for preventing further hypoglycaemic episodes was also analysed. The study included both type 1 and type 2 patients.

First review collected a sample of 17 hypoglycaemic patients over a week. Only $47 \%$ of patients had a repeat BM within the hour and $24 \%$ had a record in the notes of the treatment given. No patients had symptoms reported, two patients $(12 \%)$ had a possible cause of hypoglycaemia reported, and only $35 \%$ of patients had recorded notes of action taken to prevent further hypoglycaemia.

See supplementary file: ds5430.doc - "Hypoglycaemia Documentation"

\section{Design}

To enable good monitoring of inpatient hypoglycaemia to occur the establishment of simple treatment protocols has been shown to work by offering prompt recognition and action in regards to hypoglycaemic events. One such study developed a hypoglycaemic reduction bundle targeting common and simple remediable contributors to hypoglycaemia[16]. They found that the introduction of these protocols greatly reduced rates of severe hypoglycaemic events and the interventions could be transferred to other hospitals.

With this in mind this project aimed to develop a simple protocol that could be used on the receiving wards of a busy tertiary hospital with the aim to reduce instances of hypoglycaemia and improve the treatment and response to any events. Firstly, a brief meeting was had with the floor manager to help promote awareness around the staff regarding management of hypoglycaemia. This was supported by meeting staff individually to discuss identification, management, documentation, and prevention of hypoglycaemia. Following this step of creating awareness small summary sheets on management of hypoglycaemia were attached to all BM machine boxes to remind users of the steps to take upon identifying a hypoglycaemic episode. Staff were in agreement with proposed plans and with this support and increased awareness intervention was easily accomplished with placement of guidance next to the instruments used to measure BM of patients. This period of intervention was played out for one week before a further two periods of analysis post-intervention.

\section{Strategy}

The aim of this study was to analyse the management of diabetic patients experiencing hypoglycaemia in acute medical wards and compare this against recommended guidelines. A further aim was then to see if improvements in monitoring and care could be achieved via the implementation of simple interventions.

To achieve this three rounds of monitoring were conducted. In all rounds patients whom experienced hypoglycaemia were identified and then the intervention that followed was analysed. This was particularly focused around the need for repeat BM within the hour of identifying a low BM and the recording of symptoms, causes, management, and interventions taken to prevent further episodes. In between the rounds of analysis an intervention was installed. This involved speaking to ward staff members about the recommended protocols and placing a summary document of actions to be taken on discovery of a low BM next to every BM machine.

A preliminary round of analysis was conducted before the first phase of analysis to gain an appreciation of the areas that needed to be analysed. From this preliminary assessment it was predicted that the full review in phase I would identify a number of areas for improvement, however discussions with ward staff suggested basic interventions. Thus, with this preliminary data phase I, II, and III were designed with the view that good improvements could be made.

The study was carried out successfully and matched initial predictions well. A number of areas for improvement were clearly identified and improvements were made with the interventions initially designed.

Further improvements on this test could be made by increasing the length of study time and number of patients. Repeating the analysis after an extended period of time to ensure continued improvement 
would also be desirable.

\section{Results}

Post-intervention a further two rounds of data collecting was implemented. This procedure was identical to pre-intervention in that hypoglycaemic patients were identified, with the same target i.e. blood glucose level less than $4 \mathrm{mmol} / \mathrm{l}$, and their management analysed. The patients identified were again located within the medical receiving wards of Glasgow Royal Infirmary and included both type 1 and type 2 diabetes patients.

This second and third review both obtained a sample of 16 hypoglycaemic patients. On both the second and third round $100 \%$ (improvement from $47 \%$ ) of patients received a repeat BM within the hour. An improvement in phase II (69\%) and phase III (75\%) (improvement from 24\%) was seen for recording in the notes of specific treatment given. Report of symptoms remained relatively low in both phase II (25\%) and Phase III (13\%), however this shows an increased awareness of symptom documentation as preintervention no patients had symptoms recorded. Unfortunately no patients had a possible cause of hypoglycaemia reported in either phase II or III. Although, perhaps more importantly, $88 \%$ (phase II) and $94 \%$ (phase III) (improvement from $35 \%$ ) of patients now had a recorded note of action taken to prevent further hypoglycaemic episodes.

See supplementary file: ds6240.xIsx - "Data for Phase I, II \& II"

\section{Lessons and limitations}

A number of good lessons were learnt from this study despite its small size. Particularly of note is the significant impact that can be made in this area with small, targeted approaches to improvement. However, more power could be given to this with a number of improvements and amplifications to the study.

Further cycles of the project would be required to fully test the impact of the intervention and whether it could stand the test of time. Furthermore, multiple cycles would be beneficial in achieving greater outcomes and perhaps improve the recording of symptoms (a factor this study failed to achieve) which could improve the recognition of hypoglycaemic episodes in patients. Greater data could also be obtained by extending the project and acquiring a larger sample size of patients.

One limitation was the time required to look through the BM recording machines to identify patients. If a database of the data could be accessed this would significantly aide the ability to identify patients. However, the intervention itself is simple, sustainable, and has been able to make good improvements in the small size. Overcoming the challenge of collection data and extending the time length and sample size is required to test this study further.

\section{Conclusion}

This study clearly highlighted an area of inpatient diabetes management where quality improvement can be achieved with simple measures in patient care. Short conversations with staff and provision of simple guidelines on hypoglycaemia significantly improved monitoring of BM's in diabetic patients with improvements also in recording of symptoms manifested. Moreover, importantly, there was a great improvement in the recording of actions that needed to be implemented (such as increased observation) to attempt prevention of further hypoglycaemic episodes. This is a good demonstration of the simple measures that can be taken to significantly improve the care of in patients with diabetes in a busy ward environment and prevent hypoglycaemic episodes.

\section{References}

1 NHS. (2013). ThinkGlucose: Inpatient care for patients with diabetes. [Online] Available from:

http://www.institute.nhs.uk/quality and value/think glucose/welcom e to the website for thinkglucose.html.

2 NICE [2015]. NICE Guidelines.[Online] Available from: http://pathways.nice.org.uk/pathways/diabetes/ongoing-care-for-adu Its-with-type-1-diabetes\#content=view-quality-statement\%3Aqualitystatements-inpatient-care

3 Clement S, Braithwaite SS, Magee MF, Ahmann A, Smith EP, Schafer RG, Hirsh IB. (2004) Management of diabetes and hyperglycaemia in hospitals. Diabetes Care. 27: 553-591.

4 The DCCT Research Group: The effeact of intensive treatment of diabetes on the development and progression of long-term complications in insulin dependent diabetes mellitus. New Engl J Med 329:977 -985, 1993.

5 Kagansky N, Levy S, Brelje T. Hypoglycaemia as a predictor of mortality in hospitalised patients. Archives Internal Medicine. 2003; 163: $1825-1829$

6 Shorri RI, Ray WA, Daugherty JR, Griffin MR. Incidence and risk factors for serious hypoglycaemias in older persons using insulin or sulphonylureas. Archives of Internal Medicine. 1997; 157; 1681 . 1686.

7 Stagnaro-Green A, Barton MK, Linekin PI, Corkery E, deBeer K, Roman SH. Mortality in hospitalised patients with hypoglycaemia and sever hyperglycaemia. Mount Sinai Journal of Medicine 1995; 62:422-426.

8 J Diabetes Complications. (2014) Jul-Aug;28(4):565-72. doi: 10.1016/j.jdiacomp.2014.03.002. Epub 2014 Mar 10.

9 Deepak PJ, Suitha K, Nagaraj J, Sanjukta A, Bhattacharrya A. Inpatient management of diabetes: survey in a tertiary care centre. Postgraduate Medical Journal. 2003; 79:585-587.

10 Zapatero A et al. Frequency of hypoglycaemia and its impact on length of stay, mortality and short-term readmission in patients with diabetes hospitalised in internal medicine wards. Endocr Pract. 2014; 20(9):870-5. 
11 Metchich LN, Petit WA, Inzucchi SE. The most common type of hypoglycemia is insulin-induced hypoglycemia in diabetes. Am J Med. 2002; $113:$ 317-323.

12 Goetz CG. Textbook of Clinical Neurology. 2nd ed. Philadelphia. Elsevier. 2003; p.822 -823

13 NHS Salford 2006. Clinical Management of Hypoglycaemia.

[Onlline] Available from:

http://www.salforddiabetescare.co.uk/admin/resources/uploaded/Hy poglycaemia\%20Policy40.pdf

14 Diabetes UK 2015. [Online] Available from:

http://www.diabetes.org.uk/Guide-to-diabetes/Complications/HyposHypers/

15 Diabetes UK 2015. Hypos And Hypers. [Online] Available from: http://www.diabetes.org.uk/Guide-to-diabetes/Complications/HyposHypers/

16 Maynard $\mathrm{G}$ et al. Impact of a hypoglycaemia reduction bundle and a systems approach to inpatient glycaemic management. Endocr Pract. 2015; 2;21(4):355-67.

\section{Declaration of interests}

Nothing to declare.

\section{Acknowledgements}

Nil

\section{Ethical approval}

Improvement study with simple monitoring of patient management. No direct intervention on patients. 